\title{
AUTOMATIC PROSODY BOUNDARY LABELING OF MANDARIN USING BOTH TEXT AND ACOUSTIC INFORMATION
}

\author{
Chongjia $\mathrm{Ni}^{1,2}$, Wenju $\mathrm{Liu}^{l}$, and Bo Xu ${ }^{l}$ \\ ${ }^{1}$ National Laboratory of Pattern Recognition, Institute of Automation, \\ Chinese Academy of Sciences, Beijing 100190 \\ ${ }^{2}$ School of Statistics and Mathematics, Shandong University of Finance, Jinan 250014 \\ \{cjni, lwj, xubo\}@nlpr.ia.ac.cn
}

\begin{abstract}
Prosody is an important factor for a high quality text-tospeech (TTS) system. Prosody is often described with a hierarchical structure. So the generation of the hierarchical prosody structure is very important both in the corpus building and the real-time text analysis, but the prosody labeling procedure is laborious and time consuming. In this paper, an automatic prosody boundary label system is presented, in which the classification and regression tree (CART) framework is used. In this system, we build a prosody model using acoustic information and the text information based on large speech corpus with prosodic structure label (ASCCD). Experiments show this model can achieve prosody boundary detection $90.86 \%$ accuracy.
\end{abstract}

Index Terms - prosody boundary, CART, Chinese information processing, prosody prediction, acousticprosodic feature

\section{INTRODUCTION}

Prosody is generally used to describe aspects of a spoken utterance's pronunciation which are not adequately explained by segmental acoustic correlates of sound units (phones). The prosodic information associated with a unit a speech, syllable, word, phrase, or clause, influences all the segments of the unit in an utterance. They are also referred to as supra-segmental that transcend the properties of local phonetic context.

Prosody encoded in the form of intonation, rhythm and accent conveys linguistic and paralinguistic information such as emphasis, intent, attitude and emotion of the speaker. Prosody is also used by speakers to provide cues to the listener and aid in appropriate interpretation of their speech. Prosodic phrase boundary location is a basic problem in the field of prosody research. Prosody in spoken language correlates with acoustic and syntactic features, such as duration, accent and pitch. It can be beneficial in speech applications such as TTS, automatic speech recognition (ASR) and natural language understanding etc. In order to integrate prosody to these applications, it is important to represent prosody suitably. There are distinct prosody label systems, such as Tones and Break Indices (ToBI) [1], TILT intonational model[2], Fujisaki model[3] Intonational Variation in English (IViE)[4]. And to mandarin, there is CToBI prosody label system [5]. These prosodic labeling approaches provide a common framework for charactering prosody. However, the manually prosody labeling is laborious and time-consuming. Moreover, to keep the consistence between different labelers and even the same labeler in different time is some difficult. So automatic prosody labeling attracts more and more attention now. Some research work had been done in this field [6-10]. S.Narayanan[6] applied acoustic and syntactic features in maximum entropy framework to label prosody boundary and accent automatically. M.Ostendorf[7] used decision trees and a Markov sequence model to predict the prosody boundary of the text. $\mathrm{Ma}[8]$ applied decision tree to label prosody boundary using both text and acoustic information. S.Ananthakrishnan[9] use a coupled Hidden Markov Model to recognize the prosody boundary and accent through using acoustic lexical and syntactic information. $\mathrm{Hu}[10]$ used CART to classify the prosodic type. In this paper, an automatic prosody labeling method is proposed that attempts to speed up the corpus building process.

The paper is organized as follows. In section 2 , prosody labeled corpus is introduced and a prosodic structure is described. In section 3 , the acoustic feature of the prosodic phrase boundary is analyzed. In section 4 , the prosody labeling algorithm is presented. The experiment will be reported in section 5. And the conclusion will be drawn finally.

\section{ASCCD MANDARIN CORPUS}

A large mandarin speech corpus, ASCCD, designed for TTS and labeled with prosodic ties, is used in our search. The text of ASCCD contains 18 pieces of narration or argumentum. Each piece contains 2 5 sections and 500 600 syllables. The text was read by 10 speakers (five male and five female). The speech was labeled with syllable in SMAPLA-C system and labeled prosody accent, boundary and tone in $\mathrm{C}-\mathrm{ToBI}$ system [5]. In the corpus, prosodic 
boundary was labeled by $0,1,2,3,4$, which stand for syllable boundary in prosody word, prosody word boundary, minor prosody phrase boundary, major prosody phrase boundary and intonation group boundary respectively. Different type of breaks is corresponding to different type of prosody boundary. Break0 (Bk0), Break1 (Bk1), Break2 (Bk2), Break3 (Bk3), Break4 (Bk4) are the syllable boundary in prosody word, prosody word boundary, minor phrase boundary, major phrase boundary and intonational group boundary respectively. Table 1 lists the distribution of different type of breaks in ASCCD.

Table 1 Break distribution in ASCCD.

\begin{tabular}{|c|c|c|c|c|c|}
\hline Total & Bk0 & Bk1 & Bk2 & Bk3 & Bk4 \\
\hline 87628 & 54393 & 14799 & 7730 & 6996 & 3710 \\
\hline $100 \%$ & $62.1 \%$ & $16.9 \%$ & $8.8 \%$ & $8.0 \%$ & $4.2 \%$ \\
\hline
\end{tabular}

We describe the prosody structure into 5 hierarchical layers: Phone foot (PF), Prosodic word (PW), Minor phrase (MIP), Major phrase (MAP) and Intonation Group (IG).Their relationships are showed in the Fig. 1.

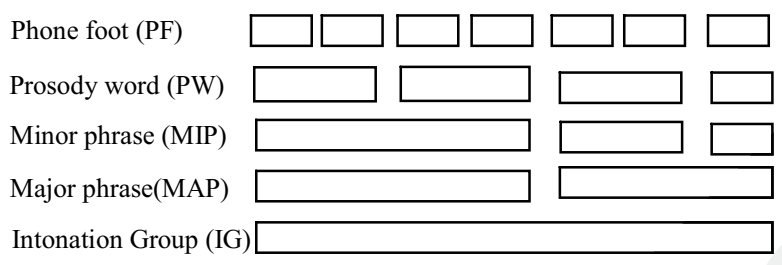

Fig. 1 Prosody Structure

\section{ACOUSTIC FEATURE OF THE PROSODY PHRASE BOUNDARY}

\subsection{Acoustic features}

Recently, the research on mandarin prosody phrase boundary location is mainly for the purpose of TTS and automatic prosody labeling. They hope to start from text and acoustic information, employ statistical method or knowledge inducted method, and get the prosody characteristic of spoken language, so they can get the prosody boundary [6-10]. At the same time, based on large prosody labeled speech corpus, many researchers start to study the acoustic parameter of prosody structure. They hope to get more knowledge about the acoustic feature of prosody structure. Liu [11] analyzed the acoustic features of prosody at all levels in Chinese spontaneous speech using statistical method in order to find the cues of prosody phrase boundary in spontaneous speech. He found that pause, preboundary syllable lengthening, F0 reset and F0 range are major cues of boundaries in Chinese spontaneous speech. Lin [12] showed the relation between breaks and prosody structure. He pointed out that there are two types of break that can be apperceived in mandarin speech: break with silent pause (SP) and break with filled pause (FP). In general, the higher the boundary level is, the higher of the percentages of number of SPs is. F0 reset and F0 range distinguish between minor and major break.

We can also employ the speech analysis tool, Praat (by Paul) to study the prosody feature of the speech visually (Fig. 2). From the figure, we can see the F0 reset, the duration of syllable prolong, and the pause occur at breaks.

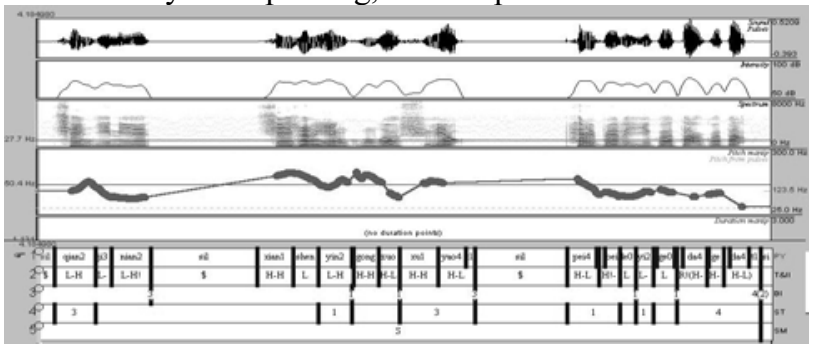

Fig.2 Speech analysis by Praat

According to above cues of prosody boundary (PB) in mandarin, we present a serial of parameters as illustrated in table 2 .

Table 2 List of some acoustic parameter of PB.

\begin{tabular}{|l|l|}
\hline Parameter name & Interpretation \\
\hline SilD & Silence duration \\
\hline DurS & $\begin{array}{l}\text { The difference between the duration } \\
\text { of syllable before break and the } \\
\text { followed syllable }\end{array}$ \\
\hline PDur & The duration of pitch discontinuity \\
\hline $\begin{array}{l}\text { PDlt BPDlt } \\
\text { TPDlt PMDlt }\end{array}$ & Pitch difference \\
\hline E E1 E2 & Energy \\
\hline T1 & Tone of syllable \\
\hline SNum1 & Distance from the last break before \\
\hline K1 K2 & Slope \\
\hline SNum & $\begin{array}{l}\text { Distance from the last syntax word } \\
\text { boundary before }\end{array}$ \\
\hline
\end{tabular}

\subsection{Acoustic analysis}

We calculate the features at all different type of prosody boundary. Some of them are list in table 3 .

Table 3 The mean value of some features

\begin{tabular}{|l|l|l|l|l|l|}
\hline Name & \multicolumn{1}{|c|}{ Bk0 } & Bk1 & Bk2 & Bk3 & Bk4 \\
\hline SilD & 0.011 & 0.021 & 0.095 & 0.496 & 0.884 \\
\hline DurS & 54.49 & 37.86 & 80.79 & 87.62 & 73.32 \\
\hline PDur & 1.95 & 2.357 & 11.33 & 48.79 & 87.76 \\
\hline E & 9.246 & 9.095 & 9.427 & 9.428 & 9.242 \\
\hline PDlt & 0.083 & 0.111 & -0.148 & -0.382 & -0.442 \\
\hline BPDlt & -0.133 & -0.055 & -0.059 & -0.029 & -0.162 \\
\hline TPDlt & -0.563 & -0.160 & -0.955 & -1.599 & -1.633 \\
\hline PMDlt & -0.275 & -0.068 & -0.373 & -0.604 & -0.735 \\
\hline
\end{tabular}

In table 3 , the scale for silence duration is second, and in order to eliminate the influence of different people and reduce the computing complexity, we normalized these features to a certain range. To the syllable duration, we use 
formula (1) to normalize. To pitch information (F0), we use formula (2) to normalize it.

$T=\frac{t-\mu}{\sigma}, T= \begin{cases}-100 & T<-1 \\ T^{*} 100 & -1 \leq T \leq 1 \\ 100 & T>1\end{cases}$

Where $\mu$ is the mean duration of compound vowel, $\sigma$ is the standard deviation of compound vowel, and $t$ is the compound vowel's duration of the syllable.

$$
\breve{F}=\frac{F-F_{\text {min }}}{F_{\text {max }}-F_{\text {min }}} * C
$$

Where $F$ is fundamental frequency, $F_{\min }$ is the minimal fundamental frequency, and $F_{\max }$ is the maximal fundamental frequency in speech data of a speaker, $C$ is a const.

From table 3, we can find these features different at different prosody boundary. Such as the silence duration after the boundary, and the syllable duration before the boundary are obviously different.

\section{PROSODY BOUNDARY CLASSIFICATION ALGORITHM}

We used the acoustic feature listed in table 2 and the text feature. The text information is also used, such as the Chinese word length and current position whether or not is the Chinese word boundary. The automatic Chinese word segmentation tool is used to generate segmentation information.

\subsection{CART Method}

Using acoustic features and text information, we can employ classification and regression tree (CART) to classify all these kinds of prosody boundary. CART is used here to classify a feature vector $\mathrm{x}$ by asking a series of question about the element of $x$, and finally map it to a leaf node. The CART can provide the conditional probability distribution of the type of boundary at the leaf nodes. In order to construct a CART, some basic problem should be treated: feature selection, question set designing, splitting rule and stopping criterion.

Since the feature was normalized, we need to design some logic compare questions, for example $A>x$ and so on. We use information gain to measure the entropy of expectation in order to split the node, and when the number that node holds element is smaller than a threshold value, we stop splitting the node.

\subsection{An improved model based on CART}

According to the definition of major phrase prosody and intonational group boundary, there is obviously silence after the prosody boundary. So we can use this character to classify the boundary set according to whether or not the silence duration is zero.

And we know that the minor phrase boundary is such type boundary that it is located between the prosody word boundary and major phrase boundary. And the silence after this type boundary is not obvious. If it is labeled by different people, it would lead to different result. So it is hard to classify. In order to improve the effect of classification of this type boundary, we design the following algorithm. We reclassify the first classification result labeled the Break1.

Algorithm:

Step1: According to the silence duration after the syllable whether or not it is zero, we classify the candidate boundary into $\mathrm{Cls} 1$ and $\mathrm{Cls} 2$. Cls1 denotes the silence duration is zero, and Cls 2 denotes the silence duration is not zero.

Step2: To Cls1, according to whether or not it is the Chinese word boundary, we classify the obvious the Break0. If the candidate boundary is not the syntax word boundary, we think the boundary is Break0. If the candidate boundary is the Chinese word boundary, we use CARTModell to classify the candidate boundary into one of Break0, Break1 and Break2. To the classified "Break1" by CARTModel1, we use the other CARTModel2 to reclassify it into Break1 and Break2.

Step3: To Cls2, we use CARTModel3 to classify the candidate boundary into the one of Break0, Break1, Break2, Break3 and Break4. To the classified "Break1" by CARTModel3, we use the other CARTModel4 to classify it into Break1 and Break2.

The procedure of algorithm is illustrated in figure 3 .

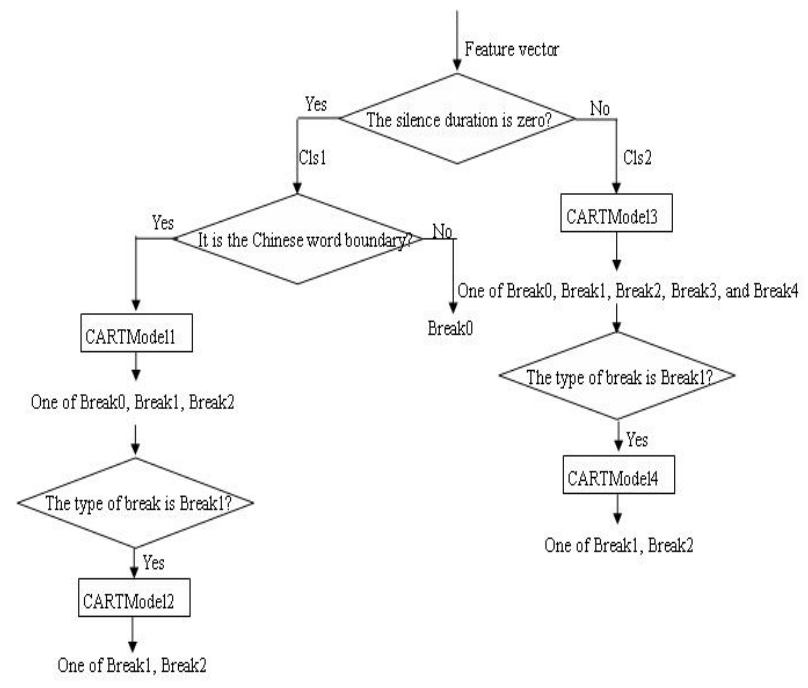

Fig. 3 Procedure of the algorithm

\section{EXPERIMENTS}

At first, we use the speech data only coming from the speaker F001. We use the data set as the train set and the 
test set to verify the classification ability of the algorithm (Inner set test). The test result listed in the table 4.

Table 4 The inner set test result of the speaker F001

\begin{tabular}{|c|c|c|c|c|c|c|}
\hline Data & Bk0 & Bk1 & Bk2 & Bk3 & Bk4 & Cor.\% \\
\hline Bk0 & 5543 & 0 & 0 & 4 & 1 & 99.91 \\
\hline Bk1 & 31 & 1532 & 29 & 0 & 0 & 96.23 \\
\hline Bk2 & 15 & 72 & 515 & 14 & 0 & 83.60 \\
\hline Bk3 & 0 & 0 & 0 & 579 & 12 & 97.97 \\
\hline Bk4 & 0 & 0 & 0 & 12 & 353 & 96.71 \\
\hline
\end{tabular}

From table 4, we know Break1 was potential classified as Break0 and Break2, the confusion among Break0, Break1, and Break2 is evident, and as well as confusion between Break 3 and Break4. And from the algorithm, we can know that the most important feature to classify the prosody boundary is the silence duration after the break, SilD.

In order to test the algorithm further, we select the first 50 sections of 10 speakers (totally 75 sections for each speaker) to composite a training set TR, and the left composite the test set $\mathrm{T}$. We use training set $\mathrm{TR}$ to train CART model, then we use the test set $\mathrm{T}$ to test the classify ability of the algorithm. The test result is list in table 5 .

Table 5 Test result by different test corpus (Cor.\%)

\begin{tabular}{|c|l|l|l|l|l|l|}
\hline Data & Bk0 & Bk1 & Bk2 & Bk3 & Bk4 & mean \\
\hline M001 & 99.98 & 90.44 & 57.02 & 75.31 & 77.78 & 91.75 \\
\hline F001 & 99.90 & 92.04 & 60.09 & 76.21 & 65.81 & 92.10 \\
\hline T & 99.66 & 88.42 & 56.20 & 75.24 & 71.53 & 90.86 \\
\hline
\end{tabular}

From table 5, we can find that:

(1) Different speaker have similar result, but different. So the normalized process is needed.

(2) From all the result, we find that Break2 is the most difficult to be classified, and the classification effect of this type boundary will influence the whole effect of this algorithm. And this type of boundary is easy classified the Break 1 and Break 3 by error.

(3) From the result of inner test (Table 4) and the result listed in Table 5, we find the classification ability drops sharply, especially in Break2. And we made statistics, and found that when the silence duration after the boundary is zero, the correct rate of Break2 only $29.76 \%$. The most Break2 is classified Break1 and Break0 by error. So in this case, improving the classification ability of Break2 is our next step work.

Compare with $\mathrm{Hu}[10]$, the correct rate of our detection about Break0, Break1 and Break3 is higher than his results, the correct rate about Break2 and Break4 is lower than his results, but our overall result is better than his. Why our result about Break2 and Break4 is worse than his? We think the reason is that the text information can improve the correct rate of Break1 and Break0, but when the silence duration is zero, CART classified Break2 into Break1 or Break0 by error because of the POS information constrains.

If we define a more rough prosody structure, $\mathrm{Bk} 1$ and $\mathrm{Bk} 2, \mathrm{Bk} 3$ and $\mathrm{Bk} 4$ are a single class respectively, we can get $\mathrm{Bk} 0,(\mathrm{Bk} 1, \mathrm{Bk} 2)$ and $(\mathrm{Bk} 3, \mathrm{Bk} 4)$ prosody structure, then we can get good result.

\section{CONCLUSION AND DISCUSSION}

In this paper, based on large speech corpus with prosody structure label, we study the acoustic feature at different prosody boundary. We use the CART to classify the prosody boundary. It will raise the prosody label work efficiency. And this is very important to aid speech recognition and speech understanding. But how to integrate the syntax knowledge to improve the correct rate of Break2 and how to integrate the prosody information to automatic speech recognition systems are still to be studied further.

\section{ACKNOWLEDGEMENT}

This work was supported in part by the China National Nature Science Foundation (No.60675026, No.60121302), 863 China National High Technology Development Project (No.20060101Z4073, No.2006AA01Z194), and the National Grand Fundamental Research 973 Program of China (No. 2004CB318105).

\section{REFERENCES}

[1] K. Silverman, etc. “ToBI: A Standard for Labeling English Prosody," Proc. ICSLP, pp. 867-870, 1992.

[2] P. Taylor, "The TILT Intonation Model," Proc. ICSLP, Vol. 4, pp.1383-1386, 1998.

[3] H. Fujisaki and K. Hirose, "Modeling the Dynamic Characteristics of Voice Fundamental Frequency with Application to Analysis and Synthesis of Intonation," Proc. 13th Int. Congr. Linguists, pp. 57-70, 1982.

[4] E. Grabe, F. Nolan, and K. Farrar, "IViE-A Comparative Transcription System for Intonational Variation in English," Proc. ICSLP, Sydney, Australia, pp. 1259-1262, 1998.

[5] C-ToBI: Prosodic Annotation system for Chinese, http://ling.cass.cn/yuyin/product/product_10.htm.

[6] V. K. Rangarajan Sridhar, etc. "Exploiting Acoustic and Syntactic Features for Automatic Prosody Labeling in a Maximum Entropy Framework", IEEE Trans. Audio, Speech, and Language Process., Vol.16, No.4, pp.797-811, 2008.

[7] C. W. Wightman and M. Ostendorf, "Automatic Labeling of Prosodic Patterns," IEEE Trans. Speech Audio Process., Vol. 2, No. 4, pp.469-481, 1994.

[8] X. Ma, W. Zhang, Q. Shi, W. Zhu, and L. Shen, "Automatic Prosody Labeling Using Both Text and Acoustic Information," Proc. ICASSP, Vol. 1, pp. 516-519, Apr. 2003.

[9] S. Ananthakrishnan and S. Narayanan, "Automatic Prosodic Envetn Detection Using Acoustic, Lexical, and Syntactic Evidence," IEEE Trans. Audio, Speech, and Language Process.,Vol.16, No.1,pp.216-228,2008.

[10] W. Hu T. Huang and B. Xu, "Study on Prosodic Boundary Location in Chinese Mandarin”.Proc.ICASSP,Vol.1, pp. I-501 - I504, 2002.

[11] Y. Liu, A. Li, "Cues of Prosodic Boundaries in Chinese Spontaneous Speech”, Proc. ICPHS2003, Barcelona, 2003.

[12] M. Lin, "Study on Breaks and Prosodic Phrase in Mandarin", Chinese Journal of linguistics, Vol.2, No.4, pp.210-217, 2000. 